\title{
Upregulation of microRNA-337 promotes the proliferation of endometrial carcinoma cells via targeting PTEN
}

\author{
YANGYANG CAI $^{1}$, TAO HE $^{1}$, LIDAN LIANG $^{1}$, XIN ZHANG $^{1}$ and HONGYING YUAN ${ }^{2}$ \\ ${ }^{1}$ Department of Gynaecology and Obstetrics, First Affiliated Hospital of Henan University of Science and Technology, \\ Luoyang, Henan 471000; ${ }^{2}$ Department of Pathogenic Biology, Henan University of Science and Technology, \\ Luoyang, Henan 471000, P.R. China
}

Received April 2, 2015; Accepted January 7, 2016

DOI: $10.3892 / \mathrm{mmr} .2016 .5134$

\begin{abstract}
Endometrial carcinoma (EC) is a common malignancy in females. MicroRNAs (miRs) are a class of non-coding RNA that regulate a wide variety of cellular processes, and are important in the development of multiple types of malignancy. In the present study, cancerous and adjacent non-cancerous normal tissue samples were collected from 24 patients diagnosed with EC. Reverse transcription quantitative polymerase chain reaction was performed on the tissue samples to determine the expression levels of six candidate miRs. These miRs have been previously reported to be differentially expressed in EC; however, the present study observed that only miR-337 was differentially expressed. In addition, the current study identified phosphatase and tensin homolog (PTEN) as a target of miR-337 using computational analysis and a luciferase assay. EC cells transfected with miR-337 mimics and anti-PTEN small interfering RNA demonstrated significantly decreased expression of PTEN, markedly increased proliferation and inhibition of cell apoptosis. The results indicate that miR-337 is oncogenic in EC cells, as it suppresses PTEN expression. This may facilitate the development of miR-based prevention or treatment strategies for EC.
\end{abstract}

\section{Introduction}

Endometrial carcinoma (EC) is a gynecological malignancy that occurs predominantly in Western countries, and is the fourth leading cancer among females worldwide $(1,2)$. The carcinogenic effect of uninhibited estrogen stimulation on the endometrium is well understood (3); however, the specific events that mediate estrogen stimulation resulting in tumor development remain to be elucidated. Previous studies have

Correspondence to: Professor Hongying Yuan, Department of Pathogenic Biology, Henan University of Science and Technology, 263 Kaiyuan Road, Luoyang, Henan 471000 P.R. China

E-mail: endometrialca@163.com

Key words: endometrial carcinoma, microRNA-337, proliferation, apoptosis, phosphatase and tensin homolog reported that expression of microRNAs (miRs) in the endometrium is affected by ovarian steroids, and by a gradual accumulation of a number of genetic abnormalities, which are hypothesized to be correlated with the carcinogenesis of EC. These abnormalities may activate oncogenes, inactivate tumor suppressor genes and, directly or indirectly, contribute to the pathogenesis or tumorigenesis of EC $(4,5)$. Therefore, miRs may be key in the carcinogenesis of EC.

miRs are a family of small non-coding RNAs (length, 19-24 nt) that post-transcriptionally modulate gene expression via perfect or imperfect base-pairing to messenger RNAs (mRNAs) (6). Hundreds of miRs are observed in a variety of animal genomes and they regulate $\leq 30 \%$ of human genes (7). Each miR exerts a regulatory effect on $\leq 200$ genes. Although further studies are required to investigate the biological functions of the majority of miRs, numerous miRs have been demonstrated to be involved in various biological processes, including cell proliferation, differentiation, metabolism and apoptosis $(8,9)$. The correlation between miRs, cancer progression and metastasis has been a topic of interest $(10,11)$. Lee et al (12) collected EC tissue samples and adjacent non-cancerous tissue samples, and performed an miR microarray assay to compare the expression profiles of the cancerous and adjacent non-cancerous tissues. Numerous miRs were identified to be differentially expressed in the cancerous tissue samples, with certain genes being upregulated and other genes being downregulated, when compared with the non-cancerous controls (12).

In the current study, six candidate miRs were selected: miR-224, miR-29a, miR-455, miR-221, miR-337 and miR-132. Reverse transcription quantitative polymerase chain reaction (RT-qPCR) was performed to compare the expression levels of the candidate miRs in cancerous EC and adjacent non-cancerous tissue samples. It was observed that only miR-337 was significantly upregulated in the cancerous tissue compared with the normal control. Furthermore, the target gene of the differentially expressed miR was identified and validated, and the underlying molecular mechanism of EC cell proliferation was investigated.

\section{Materials and methods}

Patients and tissue specimens. Cancerous tissue $(\mathrm{n}=24)$ and paired adjacent non-cancerous tissue $(n=24)$ samples, 


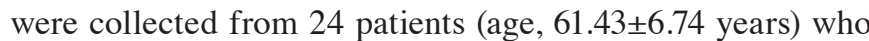
underwent a hysterectomy for the treatment of EC at the First Affiliated Hospital Of Henan University Of Science and Technology (Luoyang, China) from June 2013 to January 2015. The tissue samples were immediately frozen in liquid nitrogen following surgical removal and stored at $-80^{\circ} \mathrm{C}$. The present study was approved by the institutional review board at Henan University Of Science and Technology (Luoyang, China) and all patients provided written informed consent.

Cell lines and small interfering RNA (siRNA) transfection with miR-337 mimics and anti-PTEN. AN3-CA and HEC-1-A human EC cell lines were provided by the American Type Culture Collection (Manassas, VA, USA). AN3-CA and HEC-1-A cells were maintained at $37^{\circ} \mathrm{C}$ in RPMI-1640 medium containing penicillin $(100 \mathrm{U} / \mathrm{ml}), 15 \%$ fetal bovine serum and streptomycin $(100 \mu \mathrm{g} / \mathrm{ml})$ (all Invitrogen; Thermo Fisher Scientific, Inc.) in an atmosphere containing 5\% $\mathrm{CO}_{2}$ to no more than 10 passages. The miR-337 mimics and anti-PTEN siRNA (Ambion; Thermo Fisher Scientific, Inc.) were stored in $50 \mu \mathrm{l}$ RNase-free water $(\mathrm{pH} 7.4)$ prior to use. When cells reached a confluence of $\sim 75 \%$, Lipofectamine 2000 (Thermo Fisher Scientific, Inc.) was used for siRNA transfection. A $4 \mu 1$ volume of Lipofectamine 2000 mixed with the constructs, as above (final concentration, $50 \mathrm{nM}$ ), added onto the cultured cells and mixed. After $12 \mathrm{~h}$, the medium was replaced with fresh medium. A scrambled control, a sequence with no known target in human genome, acted as a negative control.

Western blot analysis. The M-Per Mammalian Protein Extraction reagent (Pierce Biotechnology; Thermo Fisher Scientific, Inc., Waltham, MA, USA) was used to collect whole-cell lysates from homogenized tissues or transfected cells. Following separation on $10 \%$ sodium dodecyl sulfate-polyacrylamide gels by electrophoresis $(1 \mathrm{~h}, 100 \mathrm{~V})$, proteins were transferred to nitrocellulose membranes (EMD Millipore, Billerica, MA, USA), prior to being treated with antibodies. The primary antibodies used in the present study included mouse monoclonal anti- $\beta$-actin (cat. no. sc-47778; Santa Cruz Biotechnology, Inc., Dallas, TX, USA) and mouse monoclonal anti-phosphatase and tensin homolog (PTEN; cat. no. sc-7974; Santa Cruz Biotechnology, Inc.), added for $2 \mathrm{~h}$ at room temperature. The secondary antibody used was phycoerythrin-conjugated goat anti-mouse (cat. no sc-3764; Santa Cruz Biotechnology, Inc.), and was added at $4^{\circ} \mathrm{C}$ overnight The primary and secondary antibodies used in the study were diluted at 1:1,000 and 1:10,000, respectively. Following this, the antigen-antibody complexes were visualized using enhanced chemiluminescence (GE Healthcare Life Sciences, Chalfont, UK) on X-ray film, and ImageJ software version 1.48 (www.imagej.nih.gov/ij) was used to analyze these.

$R N A$ isolation and RT-qPCR. The tissue or transfected cell samples were digested with proteinase K (Sigma-Aldrich, St. Louis, MO, USA), and TRIzol reagent (Invitrogen; Thermo Fisher Scientific, Inc.) was used to isolate the total RNA. RT-qPCR was used for analysis of miR and mRNA expression levels. cDNA was reverse transcribed from total RNA using a small RNA-specific, stem-loop RT primer from the $\operatorname{TaqMan}^{\circledR}$ Small RNA assay and reagents from the TaqMan ${ }^{\circledR}$ MicroRNA Reverse Transcription kit (Applied Biosystems; Thermo Fisher Scientific, Inc.), in accordance with the manufacturer's protocol. qPCR was performed using the TaqMan ${ }^{\circledR}$ Gene Expression assay on an Applied Biosystems ${ }^{\circledR} 7500$ Real-Time system (ABI 7500HT instrument; Applied Biosystems; Thermo Fisher Scientific, Inc.), with cycling conditions as follows: A 2-min hold at $50^{\circ} \mathrm{C}$, a $10-\mathrm{sec}$ hold at $95^{\circ} \mathrm{C}$, then 40 cycles of $15 \mathrm{sec}$ at $95^{\circ} \mathrm{C}$ and $60 \mathrm{sec}$ at $60^{\circ} \mathrm{C}$. DNase $(0.5 \mu \mathrm{l})$, primers $(10 \mathrm{pmol})$, Taq polymerase $(1 \mu \mathrm{l})$, dNTPs $(50 \mathrm{pmol})$ and 10X TaqMan Mastermix buffer solution were all sourced from Sangon Biotech Co., Ltd. (Shanghai, China). The primer sequences were as follows: miR-337 forward, 5'-ACA CTCCAGCTGGGTCAAGAGCAAT-3', and reverse, 5'-CTC AACTGGTGTCGTGGA-3'; PTEN forward, 5'-CCAGTG GCACTGTTGTTTCACA-3', and reverse, 5'-CAGGTAACG GCTGAGGGAGCTC-3'; and U6 forward, 5'-CTCGCTTCG GCAGCACA-3', and reverse, 5'-AACGCTTCACGAATT TGCGT-3'. Reverse transcriptase-free samples were used to act as negative controls. Samples were subsequently run on agarose gels for verification of product. Relative expression of samples to the reference gene RNU44 was calculated using the $\Delta \Delta \mathrm{Cq}$ method (13).

MTT assay. The 2-(4,5-dimethyltriazol-2yl)-2,5-diphenyltetrazolium bromide (MTT; Sigma-Aldrich) colorimetric assay was used to determine cell viability in cells transfected with miR-337 mimics and anti-PTEN siRNA. Following the addition of $5 \mathrm{mg} / \mathrm{ml}$ MTT solution to each well, the cells (80\% confluence; $n=3$ wells per experimental group) were incubated for $4 \mathrm{~h}$ at $37^{\circ} \mathrm{C}$. MTT crystals were dissolved in dimethyl sulfoxide and the absorbance was determined at a wavelength of $570 \mathrm{~nm}$ using a Synergy Neo2 Multi-Mode microplate spectrophotometer (BioTek Instruments, Inc., Winooski, VT, USA).

Dual-luciferase reporter assay. The full length of the 3'-untranslated region (UTR) of PTEN, which contained the putative binding sequences for miR-337, was synthesized and cloned into the firefly luciferase pGL3-control vector (Invitrogen; Thermo Fisher Scientific, Inc.). AN3-CA and HEC-1-A cells (in logarithmic growth phase) at a density of 8,000 cells/well were incubated in 24 -well plates. Following transfection with pGL3-PTEN $\left(24 \mathrm{~h} ; 37^{\circ} \mathrm{C}\right)$, co-transfection was conducted with $50 \mathrm{nM}$ negative control or miR-337, and 4 ng pRL-TK vector (Invitrogen; Thermo Fisher Scientific, Inc.) using $4 \mu \mathrm{l}$ Lipofectamine 2000 and $500 \mathrm{ng}$ of each construct, according to the manufacturer's protocols. The dual-luciferase reporter assay system (Promega Corporation, Madison, WI, USA) was conducted to determine the firefly and Renilla luciferase activities.

Apoptosis assay. The apoptosis analysis was performed using flow cytometry of transfected AN3-CA and HEC-1-A cells. Following transfection (48 h), the cells were harvested and resuspended in phosphate-buffered saline (PBS) and blocked in ethanol at room temperature overnight. EC cells were incubated with $2.5 \% \mathrm{H}_{2} \mathrm{O}_{2}$ (Sigma-Aldrich) for $2 \mathrm{~h}$ at $37^{\circ} \mathrm{C}$ prior to analysis. Following three washes with PBS, the cells were resuspended in staining solution containing $1 \mathrm{mg} / \mathrm{ml} \mathrm{RNase} \mathrm{A,} 50 \mathrm{mg} / \mathrm{ml}$ 


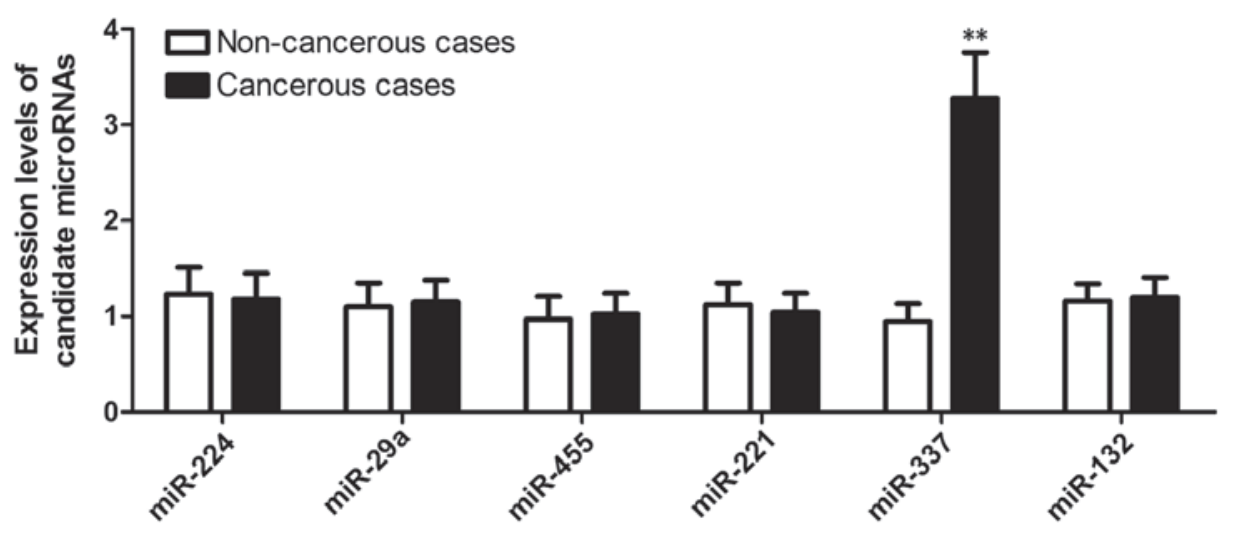

Figure 1. Expression levels of six miRs, previously reported to be differentially expressed in endometrial carcinoma, were analyzed in 24 pairs of cancerous and adjacent non-cancerous tissue samples. Only miR-337 was significantly upregulated in cancerous tissue; ${ }^{* *} \mathrm{P}<0.01$ vs. non-cancerous cases. miR, microRNA.

propidium iodide and $0.1 \%$ Triton X-100 in PBS (all obtained from Invitrogen; Thermo Fisher Scientific, Inc.). The apoptosis of stained cells was then analyzed using a FACSCalibur Flow Cytometer (BD Biosciences, San Jose, CA, USA).

Statistical analysis. GraphPad Prism software 5.0 (GraphPad Software, Inc., La Jolla, CA, USA) was used for all statistical analyses. The data was analyzed using Student's t-test or one-way analysis of variance. $\mathrm{P}<0.05$ was considered to indicate a statistically significant difference. All experiments were performed in triplicate.

\section{Results}

miR-337 is overexpressed in EC tissues. To identify the effect of miRs on the development of EC, cancerous and adjacent non-cancerous samples were obtained from 24 patients diagnosed with EC, who received surgical intervention at the First Affiliated Hospital Of Henan University Of Science and Technology. RT-qPCR was performed on the tissue samples to determine the expression of six candidate miRs, which have been reported to be differentially expressed in EC tissue (12); the current study observed that of the six miRs only miR-337 was differentially expressed (Fig. 1; $\mathrm{P}<0.01$ ).

PTEN is a target of miR-337. In silico analysis identified the tumor suppressor gene, PTEN as a potential target of miR-337 (Fig. 2A). A dual luciferase reporter assay was conducted to investigate whether the 3'-UTR of PTEN mRNA is a direct target of miR-337. The luciferase activity of miR-337-overexpressing EC cells transfected with wild-type PTEN 3'-UTR was reduced by $\sim 50 \%$ when compared with cells transfected with the scramble control $(\mathrm{P}<0.01)$. The luciferase activity of those cells transfected with mutant PTEN 3'-UTR demonstrated no increase in activity (Fig. 2B; $\mathrm{P}<0.01$ ). The results of a computational analysis (www.mirdb.org/) and luciferase assay indicate that PTEN is a validated target of miR-337 in EC cells, and miR-337 reduces the expression level of PTEN by binding to the 3 '-UTR of the gene.

Determination of miR-337 and PTEN expression levels in EC tissue samples. The expression levels of miR-337 and PTEN were determined in 24 pairs of cancerous EC and adjacent
A Hsa-miR-337 targets PTEN

Hsa-miR-337

Wild-type PTEN 3' UTR 3' -CUUCUUUCCGUAGUAUAUCCUC-5' \|\|\|\| 5' -GTGTCAGATTACCAGTTATAGGAA-3'

Hsa-miR-337

Mutant PTEN 3' UTR 3' -CUUCUUUCCGUAGUAUAUCCUC-5' || || || 5' - GTGTCAGATTACCAGTATATCCTA-3'

B

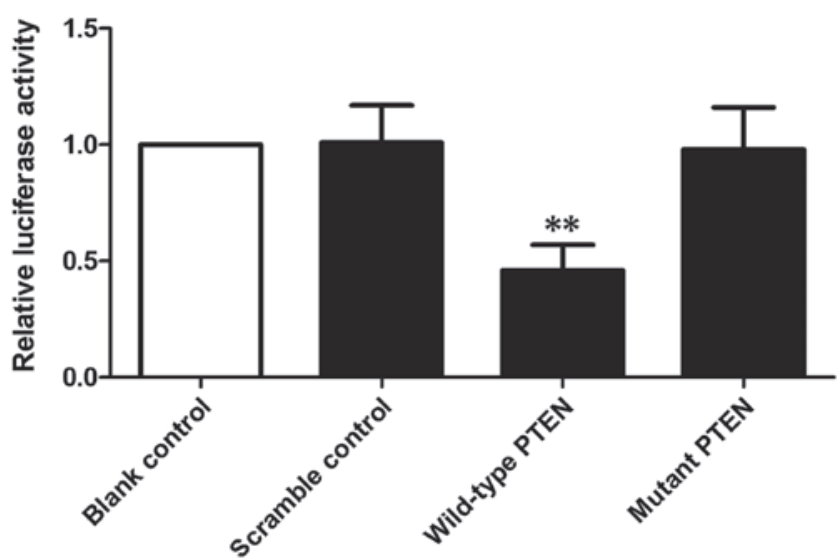

Figure 2. In silico analysis and luciferase assay. (A) Sequence comparison of miR-337 and wild-type/mutant PTEN 3'UTR. (B) Luciferase assay indicated that the miR-337-overexpressing cells transfected with wild-type PTEN 3'UTR demonstrated significantly lower luciferase activity $\left({ }^{* *} \mathrm{P}<0.01\right.$ vs. non-cancerous cases), while the luciferase activity of cells transfected with mutant PTEN 3'UTR was comparable with that of the control. miR, microRNA; PTEN, phosphatase and tensin homolog; UTR, untranslated region.

non-cancerous tissue samples, and it was observed that while miR-337 was significantly upregulated in the cancerous tissues (Fig. 3A), the mRNA (Fig. 3B) and protein (Fig. 4) expression levels of its target, PTEN, were substantially downregulated in the cancerous tissues $(\mathrm{P}<0.01)$, suggesting that PTEN is a validated target gene of miR-337 in vivo.

miR-337 regulates PTEN expression. To investigate the possible underlying molecular mechanism of miR-337 in EC, 
A

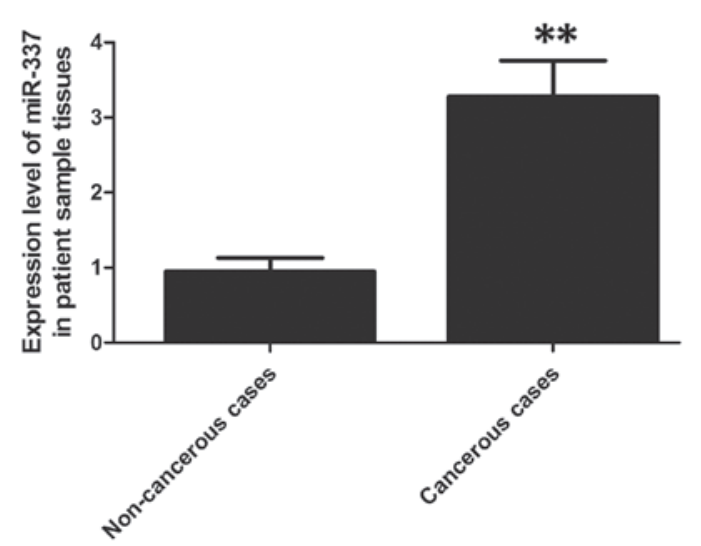

B

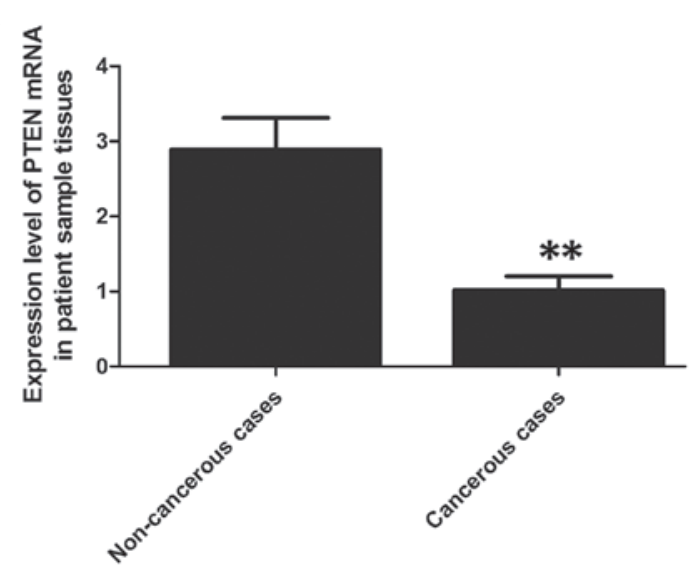

Figure 3. Determination of miR-337 and PTEN mRNA expression levels in 24 pairs of cancerous and adjacent non-cancerous tissue samples. (A) miR-337 was significantly upregulated in cancerous samples vs. the control. (B) PTEN mRNA was significantly downregulated in cancerous tissues. ${ }^{* *} \mathrm{P}<0.01$ vs. non-cancerous cases. miR, microRNA; mRNA, messenger RNA; PTEN, phosphatase and tensin homolog.

A

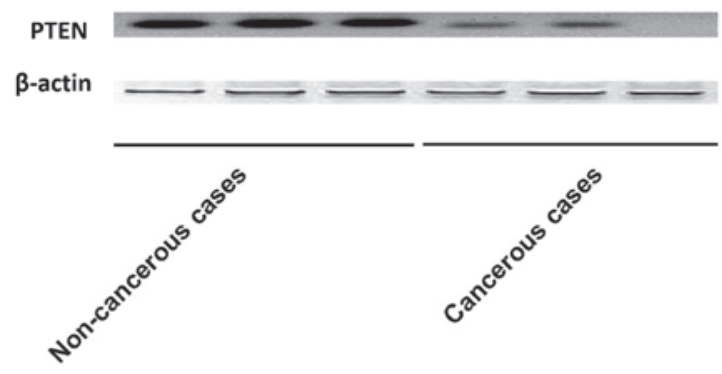

B

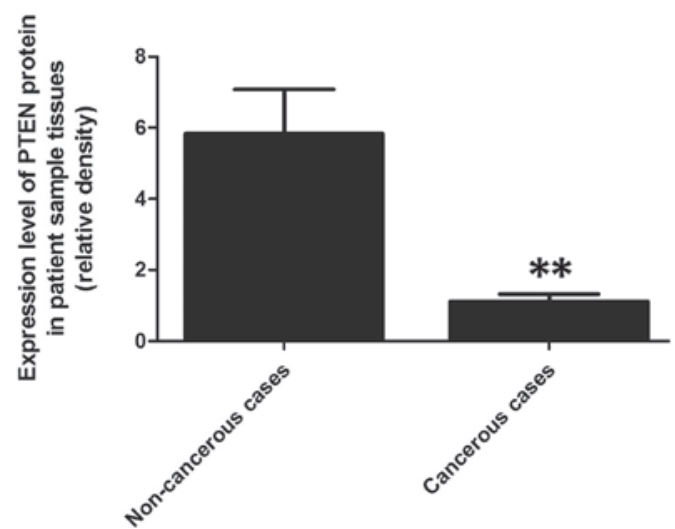

Figure 4. Determination of PTEN protein expression level in 24 pairs of cancerous and adjacent non-cancerous tissue samples. (A) PTEN protein expression levels were significantly downregulated in cancerous tissues, as determined by western blotting. (B) The relative density of the western blotting results. ${ }^{* *} \mathrm{P}<0.01$ vs. non-cancerous cases. PTEN, phosphatase and tensin homolog.

A

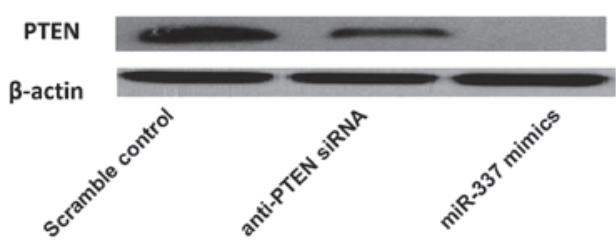

B

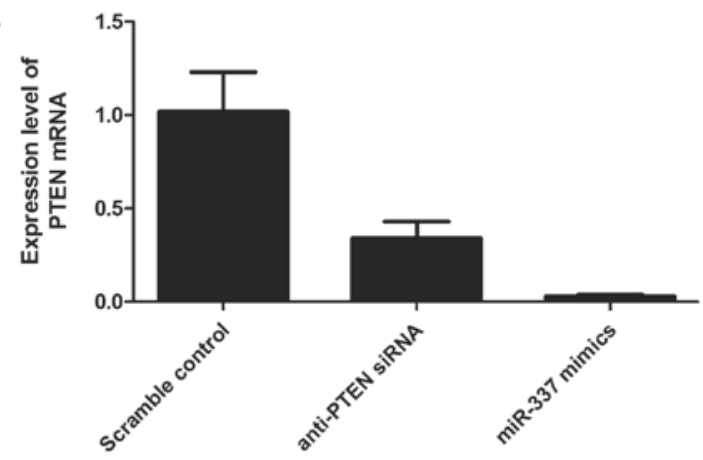

Figure 5. AN3-CA cells were transfected with scramble controls, miR-337 mimics and anti-PTEN siRNA, and the protein expression level of PTEN was determined using (A) western blotting and (B) the relative density. miR, microRNA; PTEN, phosphatase and tensin homolog; mRNA, messenger RNA; siRNA, small interfering RNA.

AN3-CA and HEC-1-A cells were transfected with miR-337 mimics to increase miR-337 expression levels. PTEN-targeting siRNA was also transfected in parallel to identify the effect on PTEN protein expression. Western blotting and RT-qPCR analyses were performed and the results demonstrated that miR-337 negatively regulated protein and mRNA expression levels of PTEN in AN3-CA (Fig. 5) and HEC-1-A (Fig. 6) cells $(\mathrm{P}<0.01)$, indicating regulation at the transcriptional level. Although the effects of miR-337 mimics and anti-PTEN siRNA on the expression of PTEN were similar in AN3-CA and HEC-1-A cells, the inhibitory effect of miR-337 was more marked than that of anti-PTEN siRNA $(\mathrm{P}<0.01)$. The results 
A

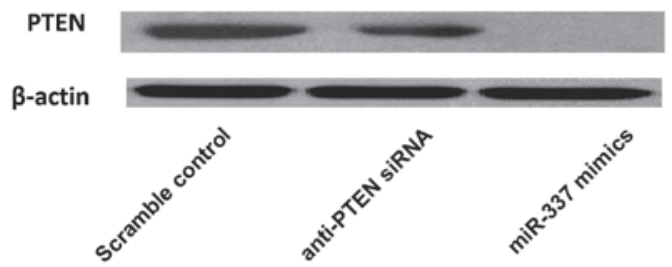

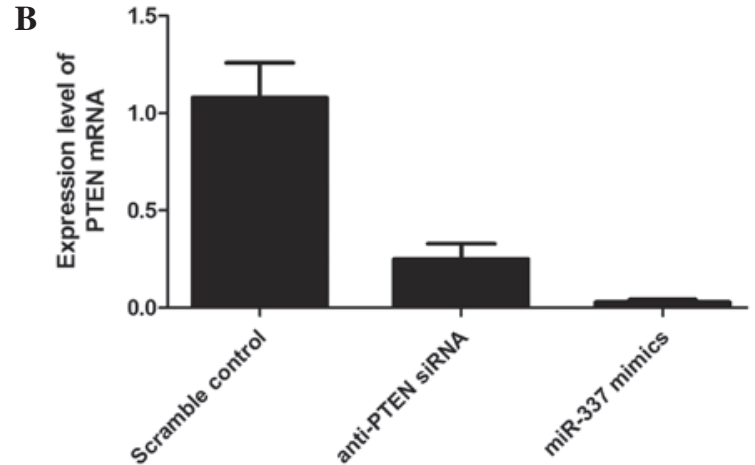

Figure 6. HEC-1-A cells were transfected with scramble controls, miR-337 mimics and anti-PTEN siRNA, and the protein expression level of PTEN was determined using (A) western blotting and (B) relative density. miR, microRNA; PTEN, phosphatase and tensin homolog; siRNA, small interfering RNA.

A

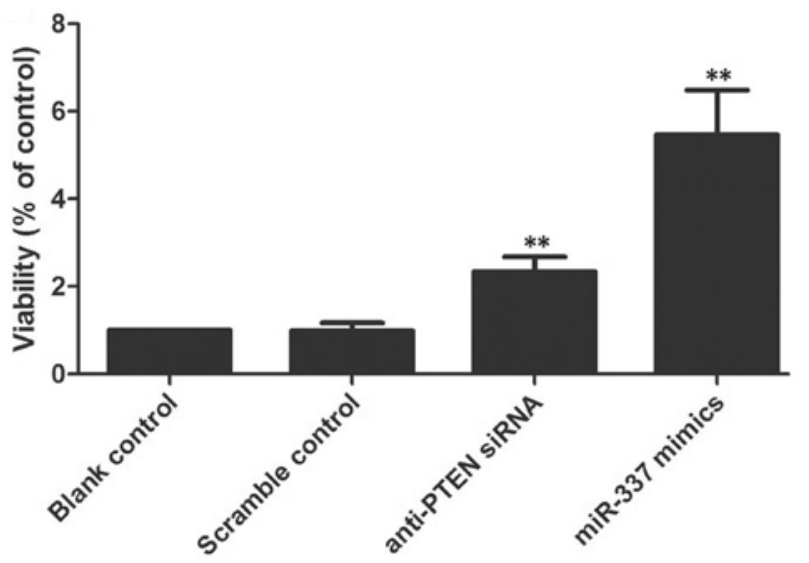

C

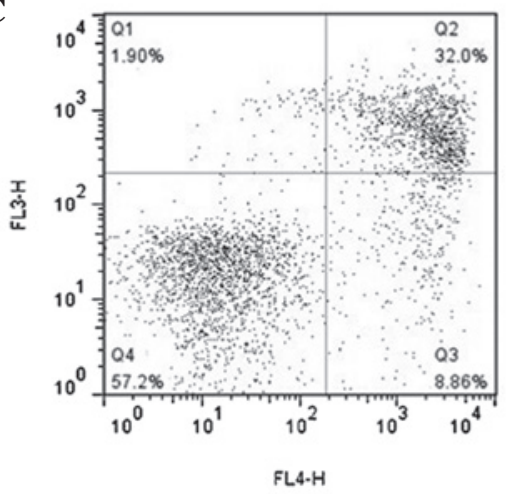

B

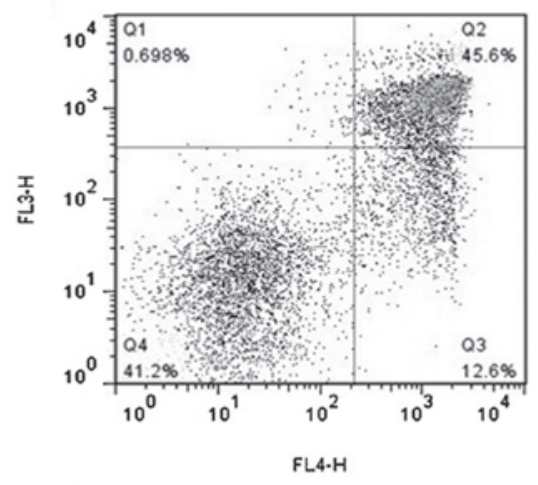

D

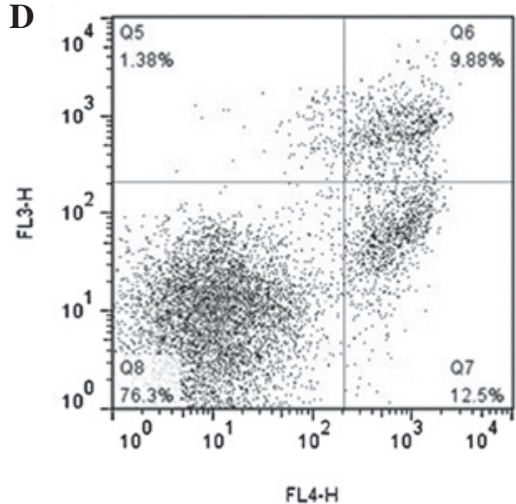

Figure 7. (A) Following transfection (48 h), the proliferation of AN3-CA cells was determined in the cells transfected with scramble controls, miR-337 mimics and anti-PTEN siRNA. The apoptotic status was determined in the cells transfected with (B) scramble controls, (C) anti-PTEN siRNA and (D) miR-337 mimics. Upper and lower quadrants indicate dead and living cells, respectively. ${ }^{* *} \mathrm{P}<0.01$ vs. scamble control. miR, microRNA; siRNA, short interfering RNA; PTEN, phosphatase and tensin homolog.

of the in vitro experiments demonstrated that miR-337 exerts a marked inhibitory effect on the expression of PTEN in EC cells $(\mathrm{P}<0.01)$.

miR-337 regulates the proliferation of EC cells. The MTT assay was used to examine the proliferative ability of EC cells. Cell proliferation was determined in AN3-CA and HEC-1-A cells at $48 \mathrm{~h}$ post-transfection, and demonstrated that the rates of cell growth in the miR-337- or anti-PTEN siRNA-transfected groups were significantly increased compared with the control groups (Figs. 7 and 8; $\mathrm{P}<0.05$ ). To investigate the underlying molecular mechanism, the apoptotic status of the differentially treated EC cells was examined. As presented in Fig. 7B-D, transfection of miR-337 and anti-PTEN siRNA significantly inhibited the apoptosis of AN3-CA cells compared with those transfected with scramble controls $(\mathrm{P}<0.01)$. Furthermore, the effects of miR-337, anti-PTEN siRNA and scramble controls on the apoptosis of the HEC-1-A cells were comparable to those observed in AN3-CA cells (Figure 8B-D; $\mathrm{P}<0.01$ ).

\section{Discussion}

Research into the underlying molecular mechanisms of EC carcinogenesis is required for the development of effective 
A

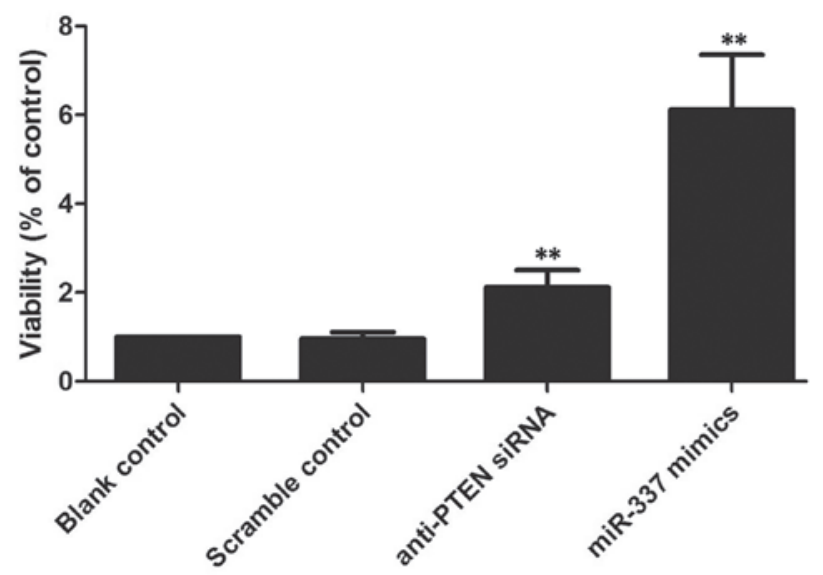

C

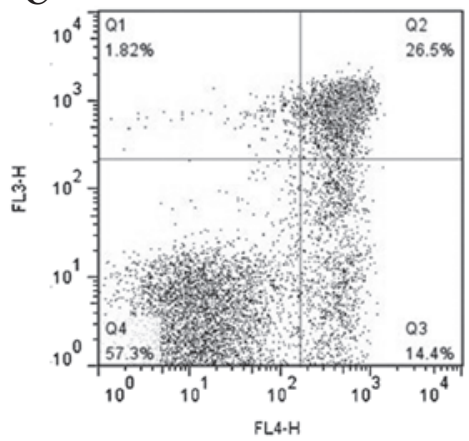

B

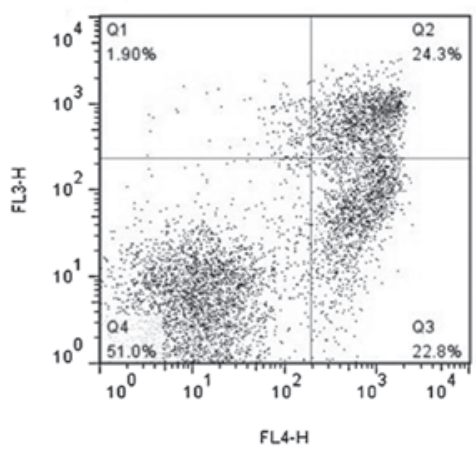

D

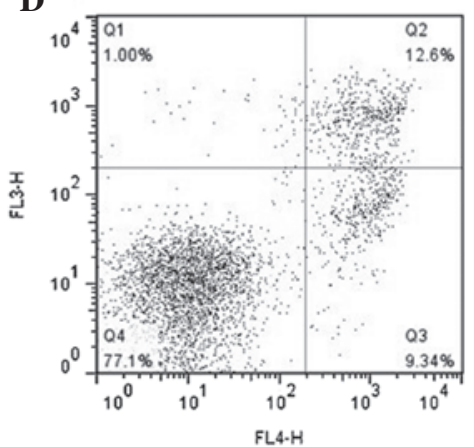

Figure 8. (A) Following transfection (48 h), the proliferation of HEC-1-A was determined in the cells transfected with scramble controls, miR-337 mimics and anti-PTEN siRNA. The apoptosis status was determined in the cells transfected with (B) scramble controls, (C) anti-PTEN siRNA and (D) miR-337 mimics. Upper and lower quadrants indicate dead and living cells, respectively. ${ }^{* *} \mathrm{P}<0.01$ vs. scamble control. miR, microRNA; siRNA, small interfering RNA; PTEN, phosphatase and tensin homolog.

strategies for diagnosis and therapy. Due to improved understanding of EC, research has expanded from tumor suppressor genes and oncogenes to include comprehensive signaling pathways and gene interaction networks (14). miRs are involved in various cellular signaling pathways, and one miR may exert an effect on numerous genes $(14,15)$. This specific characteristic of miRs has resulted in an increase in research in recent years. It is predicted that miRs modulate the expression of $30-60 \%$ of human genes $(14,16)$. Furthermore, miRs transcriptionally or translationally regulate gene expression by binding to the 3'-UTR of mRNAs (17) and miRs may act as either a tumor promoter or a suppressor, depending on the gene (18). In the present study, RT-qPCR was conducted on 24 pairs of cancerous and adjacent non-cancerous tissue samples to determine the expression of six candidate miRs, which had been previously reported to be differentially expressed in EC (13); the present study observed that only miR-337 was differentially expressed.

It has been reported that miR-337 expression levels correlate with tumor cell proliferation and tumorigenesis (19). Furthermore, its expression was observed to be associated with the tumor prognosis in certain patients (20-22). It has been determined that miR-337 is a key regulator of certain oncogenes, including homeobox C8 gene and transforming grow th factor- $\beta$ receptor type II $(23,24)$. Previous studies have demonstrated significantly reduced expression levels of miR-337 in pancreatic ductal adenocarcinoma tissue samples when compared with adjacent nonmalignant tissues (25).
Reduced production of miR-337 correlates with the lymph node status, although not with age, gender, or tumor position, diameter or differentiation $(25,26)$. However, in the present study, the upregulation of miR-337 indicates that it acts as an oncomiR and may exert an effect on a tumor suppressor. In the current study, PTEN was identified as a potential target of miR-337 using in silico analysis, which was further indicated by the results of a dual luciferase reporter assay, demonstrating that the luciferase activity of miR-337-overexpressing EC cells transfected with wild-type PTEN 3'-UTR was reduced by $\sim 50 \%$ compared with the cells transfected with the scramble control. The luciferase activity of those cells transfected with mutant PTEN 3'-UTR demonstrated no increase in activity (Fig. 2B). In addition, although miR-337 was significantly upregulated in the cancerous tissue samples (Fig. 3A), the mRNA (Fig. 3B) and protein (Fig. 4A and B) expression levels of its target, PTEN, were markedly downregulated in the cancerous tissue samples. Furthermore, the EC cell lines, AN3-CA and HEC-1-A, were transfected with miR-337 and anti-PTEN siRNAs, and the mRNA and protein expression levels of PTEN were significantly downregulated in the two cell lines, which further indicated a regulatory association between miR-337 and PTEN in EC.

The PTEN gene, at 10q23.3, encodes a dual-specificity phosphatase with lipid and protein phosphatase activities. PTEN dephosphorylates phosphatidylinositol $(3,4,5)$-trisphosphate, an important activator of Akt (27), and controls numerous 
cellular processes, including survival, cell cycle progression, metabolism and angiogenesis (28). Suppression of PTEN results in increased levels of activated phosphorylated-Akt (29). Furthermore, the reduced expression of PTEN in malignancies is associated with improved prognosis $(30,31)$. Transfection with PTEN expression vectors facilitates the proliferation of malignant cells in vitro and in vivo (32). Furthermore, frequent mutation of PTEN or loss of heterozygosity occurs in multiple types of malignancy, but rarely in EC (33). Previous studies have indicated that certain miRs exert a direct effect on PTEN (27). miR-21 was one of the earliest miRs in which its inhibition was demonstrated to elevate PTEN levels and reduce tumor cell proliferation, invasion and migration in human hepatocellular cancer (34). Later studies suggested that PTEN may be a target of miR-22, miR-221/222 and miR-144 in different types of malignancy (35-37). In the present study, the binding of miR-337 to 3'-UTR of PTEN mRNA downregulated PTEN mRNA and protein expression levels. In addition, the rates of cell growth in the miR-337- or anti-PTEN siRNA-transfected EC cells were significantly increased compared with the control, and transfection significantly inhibited apoptosis compared with the EC cells transfected with scramble controls.

In conclusion, the current study observed overexpression of miR-337 in EC tissues. As a target of miR-337, PTEN regulates expression at the transcriptional level and the inhibition of PTEN promotes the proliferation of EC cells in vitro. The findings of the present study indicate that miR-337 may be a novel target for development of miR-based prevention or treatment strategies for EC. However, the small clinical sample size was a limitation of the current study, as well as the lack of in vivo experiments investigating the function of miR-337 in EC. To further understand the role of miR-337 in the carcinogenesis of EC, studies conducted with a larger sample size across different pathological grades, and also in animal models, are required to investigate the expression levels of miR-337.

\section{References}

1. Jemal A, Ward E and Thun MJ: Recent trends in breast cancer incidence rates by age and tumor characteristics among U.S women. Breast Cancer Res 9: R28, 2007.

2. Kang S, Lee JM, Lee JK, Kim JW, Cho CH, Kim SM, Park SY, Park CY and Kim KT: How low is low enough? Evaluation of various risk-assessment models for lymph node metastasis in endometrial cancer: A Korean multicenter study. J Gynecol Oncol 23: 251-256, 2012.

3. Pecorelli S, Pasinetti B, Angioli R, Favalli G and Odicino F: Systemic therapy for gynecological neoplasms: Ovary, cervix and endometrium. Cancer Chemother Biol Response Modif 22: 515-544, 2005.

4. Wiemer EA and Berns EM: MicroRNA regulation of RAD51 in serous ovarian cancer modulates chemotherapy response. J Natl Cancer Inst 107, 2015.

5. Chen X, Yan Q, Li S, Zhou L, Yang H, Yang Y, Liu X and Wan X: Expression of the tumor suppressor miR-206 is associated with cellular proliferative inhibition and impairs invasion in ER $\alpha$-positive endometrioid adenocarcinoma. Cancer Lett 314: 41-53, 2012.

6. Lagos-Quintana M, Rauhut R, Lendeckel W and Tuschl T: Identification of novel genes coding for small expressed RNAs. Science 294: 853-858, 2001.

7. Rajewsky N and Socci ND: Computational identification of microRNA targets. Dev Biol 267: 529-535, 2004.

8. Chen X: A microRNA as a translational repressor of APETALA2 in Arabidopsis flower development. Science 303: 2022-2025, 2004.
9. Poy MN, Eliasson L, Krutzfeldt J, Kuwajima S, Ma X, Macdonald PE, Pfeffer S, Tuschl T, Rajewsky N, Rorsman P and Stoffel M: A pancreatic islet-specific microRNA regulates insulin secretion. Nature 432: 226-230, 2004.

10. Zhang B, Pan X, Cobb GP and Anderson TA: microRNAs as oncogenes and tumor suppressors. Dev Biol 302: 1-12, 2007.

11. Garzon R, Fabbri M, Cimmino A, Calin GA and Croce CM: MicroRNA expression and function in cancer. Trends Mol Med 12: 580-587, 2006.

12. Lee TS, Jeon HW, Kim YB, Kim YA, Kim MA and Kang SB: Aberrant microRNA expression in endometrial carcinoma using formalin-fixed paraffin-embedded (FFPE) tissues. PLoS One 8: e81421, 2013

13. Livak KJ and Schmittgen TD: Analysis of relative gene expression data using real-time quantitative PCR and the 2(-Delta Delta C(T)) Method. Methods 25: 402-408, 2001.

14. Friedman RC, Farh KK, Burge CB and Bartel DP: Most mammalian mRNAs are conserved targets of microRNAs. Genome Res 19: 92-105, 2009.

15. Hobert O: Gene regulation by transcription factors and microRNAs. Science 319: 1785-1786, 2008.

16. Lewis BP, Burge CB and Bartel DP: Conserved seed pairing, often flanked by adenosines, indicates that thousands of human genes are microRNA targets. Cell 120: 15-20, 2005.

17. Esquela-Kerscher A and Slack FJ: Oncomirs - microRNAs with a role in cancer. Nat Rev Cancer 6: 259-269, 2006.

18. Bandyopadhyay S, Mitra R, Maulik U and Zhang MQ: Development of the human cancer microRNA network. Silence 1: 6 , 2010.

19. Plummer PN, Freeman R, Taft RJ, Vider J, Sax M, Umer BA, Gao D, Johns C, Mattick JS, Wilton SD, et al: MicroRNAs regulate tumor angiogenesis modulated by endothelial progenitor cells. Cancer Res 73: 341-352, 2013.

20. Palmieri A, Pezzetti F, Brunelli G, Martinelli M, Scapoli L, Arlotti M, Masiero E and Carinci F: Medpor regulates osteoblast's microRNAs. Biomed Mater Eng 18: 91-97, 2008.

21. Palmieri A, Pezzetti F, Spinelli G, Arlotti M, Avantaggiato A, Scarano A, Scapoli L, Zollino I and Carinci F: PerioGlas regulates osteoblast RNA interfering. J Prosthodont 17: 522-526, 2008.

22. Annalisa P, Furio P, Ilaria Z, Anna A, Luca S, Marcella M, Marzia A, Elena M and Carinci F: Anorganic bovine bone and a silicate-based synthetic bone activate different microRNAs. J Oral Sci 50: 301-307, 2008.

23. Li Y, Zhang M, Chen H, Dong Z, Ganapathy V, Thangaraju M and Huang S: Ratio of miR-196s to HOXC8 messenger RNA correlates with breast cancer cell migration and metastasis. Cancer Res 70: 7894-7904, 2010.

24. Zhong N, Sun J, Min Z, Zhao W, Zhang R, Wang W, Tian J, Tian L, Ma J, Li D, et al: MicroRNA-337 is associated with chondrogenesis through regulating TGFBR2 expression. Osteoarthritis Cartilage 20: 593-602, 2012.

25. Zhang R, Zheng S, Du Y, Wang Y, Zang W and Zhao G: Levels of HOXB7 and miR-337 in pancreatic ductal adenocarcinoma patients. Diagn Pathol 9: 61, 2014.

26. Wang Z, Wang J, Yang Y, Hao B, Wang R, Li Y and Wu Q: Loss of has-miR-337-3p expression is associated with lymph node metastasis of human gastric cancer. J Exp Clin Cancer Res 32: 76, 2013.

27. Song MS, Salmena L and Pandolfi PP: The functions and regulation of the PTEN tumour suppressor. Nat Rev Mol Cell Biol 13: 283-296, 2012.

28. Blanco-Aparicio C, Renner O, Leal JF and Carnero A: PTEN, more than the AKT pathway. Carcinogenesis 28: 1379-1386, 2007.

29. Xu M and Mo YY: The Akt-associated microRNAs. Cell Mol Life Sci 69: 3601-3612, 2012.

30. Chang D, Wang TY, Li HC, Wei JC and Song JX: Prognostic significance of PTEN expression in esophageal squamous cell carcinoma from Linzhou City, a high incidence area of northern China. Dis Esophagus 20: 491-496, 2007.

31. Tachibana M, Shibakita M, Ohno S, Kinugasa S, Yoshimura H, Ueda S, Fujii T, Rahman MA, Dhar DK and Nagasue N: Expression and prognostic significance of PTEN product protein in patients with esophageal squamous cell carcinoma. Cancer 94: 1955-1960, 2002

32. Zhou YA, Zhang T, Zhao JB, Wang XP, Jiang T, Gu ZP, Wang XN and Li XF: The adenovirus-mediated transfer of PTEN inhibits the growth of esophageal cancer cells in vitro and in vivo. Biosci Biotechnol Biochem 74: 736-740, 2010. 
33. Ding Y, Shimada Y, Kano M, Itami A, Kawabe A, Maeda M, Li Z, Hong T, Sato F, Kaganoi J and Imamura M: PTEN/MMAC1 expression in esophageal squamous cell carcinomas. Int J Oncol 17: 695-699, 2000.

34. Meng F, Henson R, Wehbe-Janek H, Ghoshal K, Jacob ST and Patel T: MicroRNA-21 regulates expression of the PTEN tumor suppressor gene in human hepatocellular cancer. Gastroenterology 133: 647-658, 2007.

35. Bar N and Dikstein R: miR-22 forms a regulatory loop in PTEN/AKT pathway and modulates signaling kinetics. PLoS One 5: e10859, 2010.
36. Garofalo M, Di Leva G, Romano G, Nuovo G, Suh SS, Ngankeu A, Taccioli C, Pichiorri F, Alder H, Secchiero P, et al: $\mathrm{miR}-221 \& 222$ regulate TRAIL resistance and enhance tumorigenicity through PTEN and TIMP3 downregulation. Cancer Cell 16: 498-509, 2009.

37. Zhang LY, Ho-Fun Lee V, Wong AM, Kwong DL, Zhu YH, Dong SS, Kong KL, Chen J, Tsao SW, Guan XY and Fu L: MicroRNA-144 promotes cell proliferation, migration and invasion in nasopharyngeal carcinoma through repression of PTEN. Carcinogenesis 34: 454-463, 2013. 